\title{
Growth Score: A single metric to define growth in 96-well phenotype assays
}

\author{
Daniel A Cuevas ${ }^{\text {Corresp., }}{ }^{1}$, Robert A Edwards ${ }^{1}$ \\ ${ }^{1}$ Computational Science Research Center, San Diego State University, San Diego, CA, USA \\ Corresponding Author: Daniel A Cuevas \\ Email address: dcuevas@sdsu.edu
}

High-throughput phenotype assays are a cornerstone of systems biology as they allow direct measurements of mutations, genes, strains, or even different genera. Highthroughput methods also require data analytic methods that reduce complex time-series data to a single numeric evaluation. Here, we present the Growth Score, an improvement on the previous Growth Level formula. There is strong correlation between Growth Score and Growth Level, but the new Growth Score contains only essential growth curve properties while the formula of the previous Growth Level was convoluted and not easily interpretable. Several programs can be used to estimate the parameters required to calculate the Growth Score metric, including our PMAnalyzer pipeline. 
1 Growth Score: a single metric to define growth in 96-well 2 phenotype assays

3 Daniel A. Cuevas ${ }^{1}$, Robert A. Edwards ${ }^{1}$

$4{ }^{1}$ Computational Science Research Center, San Diego State University, San Diego, CA, USA

5

6 Corresponding Author:

7 Daniel Cuevas

85500 Campanile Drive, San Diego, CA, 92182, USA

9 Email address: dcuevas08@gmail.com 


\section{Abstract}

11 High-throughput phenotype assays are a cornerstone of systems biology as they allow direct

12 measurements of mutations, genes, strains, or even different genera. High-throughput methods

13 also require data analytic methods that reduce complex time-series data to a single numeric

14 evaluation. Here, we present the Growth Score, an improvement on the previous Growth Level

15 formula. There is strong correlation between Growth Score and Growth Level, but the new

16 Growth Score contains only essential growth curve properties while the formula of the previous

17 Growth Level was convoluted and not easily interpretable. Several programs can be used to

18 estimate the parameters required to calculate the Growth Score metric, including our

19 PMAnalyzer pipeline (https://edwards.sdsu.edu/pmanalyzer/).

\section{Introduction}

21 Bacterial growth of homogenous cultures is commonly described through the Monod growth

22 phases (Monod, 1949). Specifically, the three major phases of growth are lag phase, where

23 growth rate is zero and bacterial density is constant at the initial measurement; exponential

24 phase, where growth rate is at its maximum value; and stationary phase, where growth rate is

25 zero and bacterial density is constant at its maximum yield. The Growth Level (GL) has been

26 used to quantitatively measure the amount of growth displayed by a bacteria liquid culture

27 (Cuevas \& Edwards, 2017). In PMAnalyzer, an automated growth curve analysis pipeline, GL is

28 calculated following the least-squares fitting of the Zwietering logistic model (Zwietering et al., 29 1990):

$$
\hat{y}=y_{0}+\frac{A-y_{0}}{1+\exp \left[\frac{4 \mu}{A}(\lambda-t)+2\right]} .
$$


31 Here, $y_{0}$ represents the starting absorbance, $\lambda$ represents the lag time, $\mu$ represents the maximum

32 growth rate, $A$ represents the biomass yield obtained during stationary phase, $t$ represents the

33 time vector, and $\hat{y}$ represents the modeled growth curve vector. Using $\hat{y}, y_{0}$, and $A$, Growth

34 Level can be calculated as

$$
G L=\frac{n}{\sum_{i}^{n} \frac{1}{x_{i}}},
$$

where $x_{i}=\left(\hat{y}_{i}-y_{0}\right)+$ amplitude $=\left(\hat{y}_{i}-y_{0}\right)+\left(A-y_{0}\right)$.

$37 G L$ is a variation on the harmonic mean where the logistic growth curve is weighted by the amount of biomass the bacteria culture attained, or the difference in bacterial density, during the course of the experiment (represented as amplitude in the $G L$ formula). This amplitude-weighted metric performs well for differentiating growing data from growth curves that display no growth.

41 The $G L$ provides threshold values that can be used to ascribe qualitative labels or classes to growth, ranging from no growth to very high growth.

There are several mathematical drawbacks of the $G L$ formula. The harmonic mean is commonly used to average values of rates; however, absorbance data are not rate measurements. In addition, using the entire growth curve in $G L$ is implicitly affected by the lag time of the bacteria that is dependent on numerous biological properties including ageing of cells, activation of enzymes, metabolic adaptation, and other regulatory mechanisms (Monod, 1949; Robinson et al., 1998; Rolfe et al., 2012). The simplicity of the three phase growth curve is lost in the $G L$ calculation.

Here, we propose a new calculation to simplify the quantitative meaning of the level of 51 growth. The Growth Score, GS, is defined by three parameters of the Zwietering (Zwietering et al., 1990) bacterial growth curve 


$$
G S=\left(A-y_{0}\right)+0.25 \mu
$$

$54 G S$ uses the starting absorbance, $y_{0}$, biomass yield, $A$, and the maximum growth rate, $\mu$, to

compute a score for a growth curve. Without any explicit dependency on the fitted values or implicit dependencies on lag time, $G S$ is clearly understood by its growth parameters. In addition, $G S$ performs similarly to $G L$, primarily because of $G L s$ strong dependency on yield in defining a quantitative measurement of growth. GS has been implemented in the PMAnalyzer pipeline (https://edwards.sdsu.edu/pmanalyzer/) and is replacing the GL metric.

\section{Simulating growth curves}

A set of 1,000 growth curves were generated using a total time $t=240$ hours (10 days) and the Zwietering logistic model. Uniformly-distributed random values were selected for each growth curve parameter. Distribution ranges for each parameter were established to portray realistic values: starting absorbance, $y_{0}=[0.05,0.10]$; lag time, $\lambda=[0,120]$; biomass yield, $A=[0.1,1.2]$; and maximum growth rate, $\mu=[A / t, 1.1 A]$. Growth rate range is limited by the biomass yield to provide a realistic result. The first value represents an organism achieving the biomass yield at the end of the experiment; when $A$ is large this simulates very slow growth. The second value represents a rate where yield is obtained within one hour; when $A$ is large this simulates very fast growth. For cases when $A$ is small, growth rate is negligible, therefore has minimal influence on $G S$.

\section{Comparison of Growth Level and Growth Score}

Using the simulated growth curves, distributions of $G L$ and $G S$ are illustrated in the Fig. 1

histograms. The range for $G L$ is much larger than $G S$. Both metrics demonstrate a slight leftskewness but $G L$ was slightly higher at 0.143 compared to $G S$ at 0.078 . The growth score should 
75 be left skewed because a low asymptote correlates with low growth rates and results in a low

76 growth score, but the reverse is not necessarily true, high growth score values may result from

77 high or low growth rates.

78 Differences in metrics are displayed in Fig. 1 with $G L$ shown along the x-axis and the corresponding $G S$ value along the y-axis, along with a linear regression line, and growth classes. A strong correlation is shown between $G L$ and $G S$ with a Pearson correlation coefficient of 0.97 (p-value < 0.001) (Fig. 1). The qualitative classes are defined in PMAnalyzer as value thresholds.

82 Given these $G L$ classes, $G S$ class thresholds are also indicated in Fig. 1 as horizontal dotted lines.

83 A total of 191 out of the 1,000 growth curves were classified differently between the two metrics, however, only 20 growth curves had a change from "-" to "+" or vice versa. to right, the curve height (biomass yield) also decreases. Interestingly while observing biomass yield, $G L$ (Fig. 2A) does not separate curves as well as $G S$ (Fig. 2B) - there is more overlap in curve height between $G L$ classes. Fig 3. comprises of average growth curves per growth class. Standard error intervals are included in graphs but are notably small. In Fig 3., the "+++" and "++" classes show separation earlier in $G S$ (Fig. 3B) than in $G L$ (Fig. 3A). growth curve parameter. The dependence on biomass yield and maximum growth rate is further

94 depicted. Again, biomass yield overlaps between classes much more in $G L$ than in $G S$. The 95 widest distribution of yield lies in the highest growth class "++++". This provides opportunity to define more classes of growth to minimize the variation here. Maximum growth rate has less

97 influence in defining higher growth classes, and lag time has no impact on growth class. 
99 Software Foundation. Python Language Reference, version 3.6. Available at

100 https://www.python.org), NumPy version 1.13.1 (http://www.numpy.org/) (Walt, Colbert \&

101 Varoquaux, 2011), pandas version 0.20.3 (http://pandas.pydata.org) (McKinney, 2010), and

102 Seaborn version 0.8.1 (http://seaborn.pydata.org/). The ggplot2 library (Wickham, 2009, p. 2) in

103 the R programming language (R Development Core Team, 2008) was used to generate growth

104 curves in Fig. 2. See Supplementary Material 1 for the Python code that generates the random

105 curves, Supplementary Material 2 for the Jupyter Notebook version, and Supplementary Material

1063 for a PDF version of the Jupyter Notebook.

107 Biomass yield has been successful in discriminating growth from no growth using the $G L$

108 formula. However, that calculation lacked direct incorporation of the maximum growth rate, a

109 useful component in measuring fitness. Intuitively, faster growth rates should indicate a bacteria

110 that is more capable of consuming nutrients and proliferating. Yet, a higher rate of growth does

111 not solely demonstrate fitness, evidenced by the abundance of slow-growing bacteria throughout

112 the environment (e.g., $r$ and $K$ growth strategies (Pianka, 1970)). Nor is it a strong feature to

113 ultimately predict larger bacterial densities. For example, two bacteria with the same phenotype

114 can reach the same density, even if one grows at half the rate of the other. Essentially, the ability

115 to reach that potential at a faster rate indicates some biological advantage but has less

116 significance than yield. In $G S$ maximum growth rate is included at an amount of $25 \%$ in order for

117 biomass accumulation to result in the primary component of growth. Faster growth rates can

118 occur within a short time frame, resulting in low yield and high rate and, therefore, causing a

119 disproportionate $G S$ if the magnitude of the growth rate was not reduced. 
121 growth in 96-well phenotypic assays. The Growth Score provides three distinct advantages over

122 other metrics used to describe growth: First, it only uses growth curve properties (yield and

123 growth rate) in its calculation, in contrast our previous Growth Level was averaged over time and

124 was thus heavily influenced by the length of the experiment. Second, Growth Score can be used

125 with results from other software that also performs growth curve modeling or parameterization

126 (DuctApe (Galardini et al., 2014), GCAT (Bukhman et al., 2015), grofit (Kahm et al., 2010;

127 Vaas et al., 2012), OmniLog Biolog Phenotype MicroArrays (Borglin et al., 2012; Vaas et al.,

128 2012) without the need for the raw data, whereas growth level would at least need lag time and

129 /or the raw spectrophotometry data. Finally, the time independence of Growth Score is also a

130 benefit over measurements like Area Under the Curve (AUC) employed by some software. AUC

131 is subject to similar biases as growth level-longer experiments directly affect how lag time and

132 growth rate mathematically influence the AUC calculation. 


\section{Figure Legends}

135 FIGURE 1. Growth Level and Growth Score correlation. The $G L$ and $G S$ values for the

1361,000 simulated growth curves. The linear relationship results in a Pearson correlation coefficient

137 of 0.98 . Distributions are plotted as marginal histograms for each metric. Point colors represent

138 the $G L$ classes whereas dotted lines represent the proposed GS lower thresholds for each colored

139 class.

140

141 FIGURE 2. Simulated growth curves. Growth curve data stratified by $G L$ and $G S$ classes.

142 Classes are organized in decreasing growth from left to right. A) $G L$ classes. B) $G S$ classes.

144 FIGURE 3. Growth curves distributions. Average growth curves colored by $G L$ and $G S$

145 classes. +/- 1 standard error intervals are drawn around the average. A) $G L$ classes. B) $G S$

146 classes.

147

148 FIGURE 4. Growth curve parameter distributions. Box plots presenting the median and

149 interquartile ranges for each simulated growth curve parameter. Data are stratified by $G L$ and $G S$

150 classes along the columns and by parameters along the rows. Plots demonstrate how each growth

151 metric contains a strong dependence on biomass yield $(A)$ and growth rate $(\mu)$. Lag time and

152 starting yield did not have strong effects on growth class. A) $G L$ classes. B) $G S$ classes.

153 


\section{References}

155 Borglin S., Joyner D., DeAngelis KM., Khudyakov J., D’haeseleer P., Joachimiak MP., Hazen T. 156 2012. Application of phenotypic microarrays to environmental microbiology. Current opinion in 157 biotechnology 23:41-48. DOI: 10.1016/j.copbio.2011.12.006.

158 Bukhman YV., DiPiazza NW., Piotrowski J., Shao J., Halstead AGW., Bui MD., Xie E., Sato

159 TK. 2015. Modeling Microbial Growth Curves with GCAT. BioEnergy Research 8:1022-1030.

160 DOI: $10.1007 / \mathrm{s} 12155-015-9584-3$.

161 Cuevas DA., Edwards RA. 2017. PMAnalyzer: a new web interface for bacterial growth curve 162 analysis. Bioinformatics 33:1905-1906. DOI: 10.1093/bioinformatics/btx084.

163 Galardini M., Mengoni A., Biondi EG., Semeraro R., Florio A., Bazzicalupo M., Benedetti A., 164 Mocali S. 2014. DuctApe: A suite for the analysis and correlation of genomic and OmniLog ${ }^{\mathrm{TM}}$

165 Phenotype Microarray data. Genomics 103:1-10. DOI: 10.1016/j.ygeno.2013.11.005.

166 Kahm M., Hasenbrink G., Lichtenberg-Fraté H., Ludwig J., Kschischo M. 2010. grofit: Fitting

167 Biological Growth Curves with R. Journal of Statistical Software 33. DOI:

168 10.18637/jss.v033.i07.

169 McKinney W. 2010. Data Structures for Statistical Computing in Python. In: Proceedings of the

170 9th Python in Science Conference. 51-56.

171 Monod J. 1949. The Growth of Bacterial Cultures. Annual Review of Microbiology 3:371-394.

172 DOI: 10.1146/annurev.mi.03.100149.002103.

173 Pianka ER. 1970. On r- and K-Selection. The American Naturalist 104:592-597. DOI:

$17410.1086 / 282697$.

175 R Development Core Team. 2008. R: A language and environment for statistical computing.

176 Robinson TP., Ocio MJ., Kaloti A., Mackey BM. 1998. The effect of the growth environment on 177 the lag phase of Listeria monocytogenes. International Journal of Food Microbiology 44:83-92.

178 DOI: 10.1016/S0168-1605(98)00120-2.

179 Rolfe MD., Rice CJ., Lucchini S., Pin C., Thompson A., Cameron ADS., Alston M., Stringer 180 MF., Betts RP., Baranyi J., Peck MW., Hinton JCD. 2012. Lag Phase Is a Distinct Growth Phase 181 That Prepares Bacteria for Exponential Growth and Involves Transient Metal Accumulation.

182 Journal of Bacteriology 194:686-701. DOI: 10.1128/JB.06112-11.

183 Vaas LAI., Sikorski J., Michael V., Göker M., Klenk H-P. 2012. Visualization and Curve-

184 Parameter Estimation Strategies for Efficient Exploration of Phenotype Microarray Kinetics.

185 PLoS ONE 7:e34846. DOI: 10.1371/journal.pone.0034846.

186 Walt S van der., Colbert SC., Varoquaux G. 2011. The NumPy Array: A Structure for Efficient 187 Numerical Computation. Comput. Sci. Eng. 13:22-30.

188 Wickham H. 2009. ggplot2: Elegant Graphics for Data Analysis. New York, NY, USA:

189 Springer-Verlag.

190 Zwietering MH., Jongenburger I., Rombouts FM., van 't Riet K. 1990. Modeling of the Bacterial

191 Growth Curve. Applied and Environmental Microbiology 56:1875-1881. 


\section{Figure 1 (on next page)}

Growth Level and Growth Score correlation.

The linear relationship results in a Pearson correlation coefficient of 0.97 . Distributions are plotted as marginal histograms for each metric. Point colors represent the GL classes whereas dotted lines represent the proposed GS lower thresholds for each colored class. 
Figure 2 (on next page)

Simulated growth curves.

Growth curve data stratified by $G L$ and $G S$ classes. Classes are organized in decreasing growth from left to right. A) GL classes. B) GS classes. 
Growth Level

PeerJ

Manuscript to be reviewed
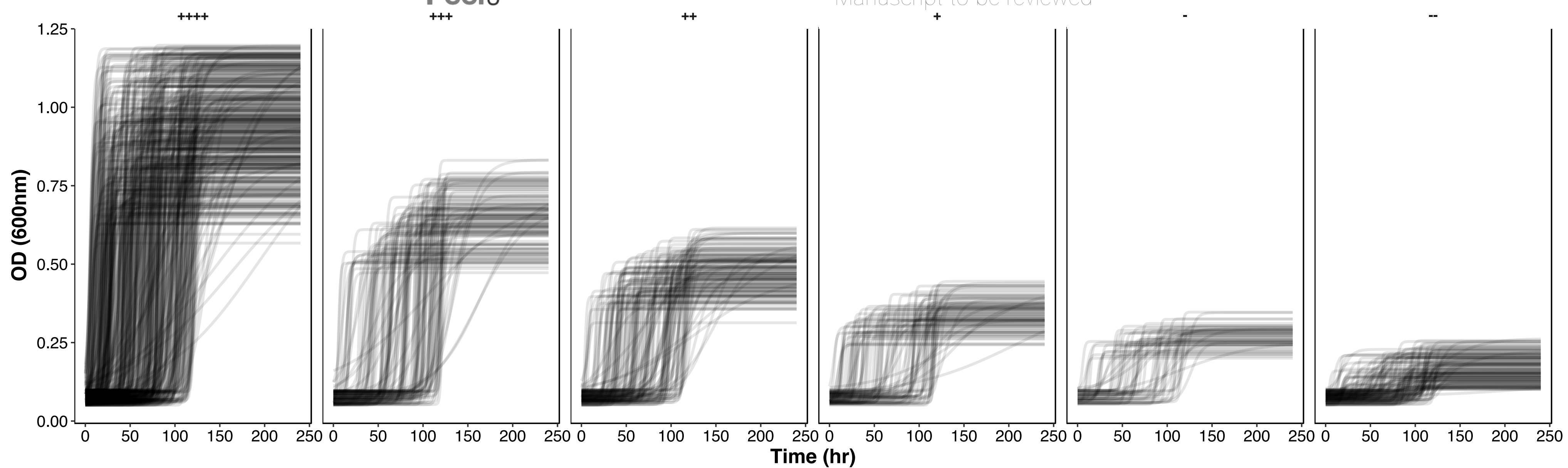

B)
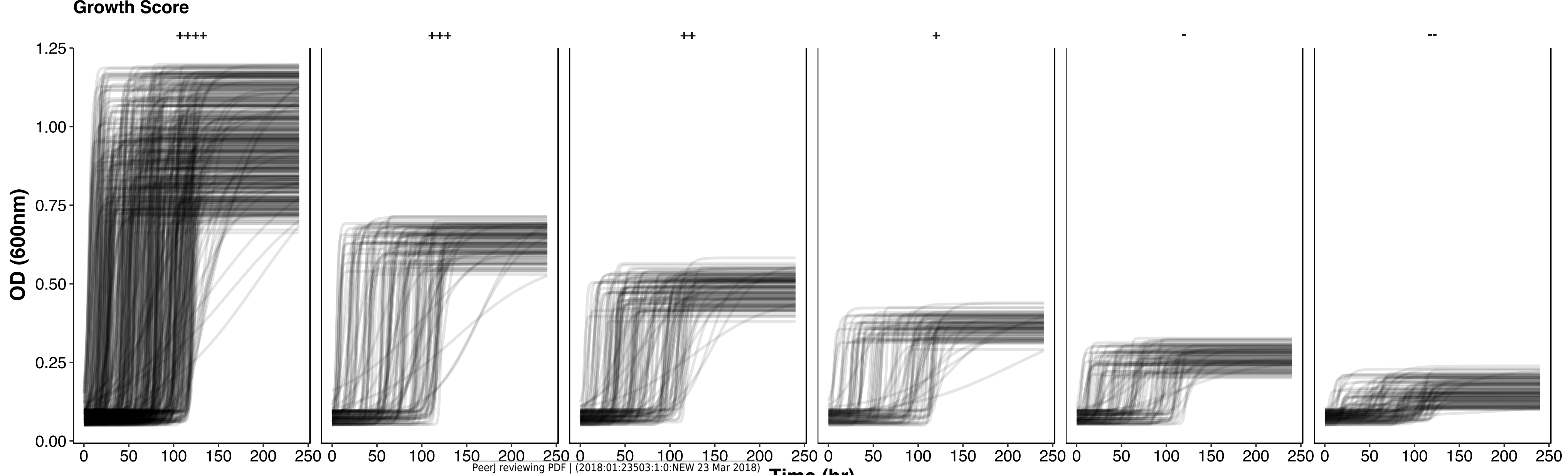

Time (hr) 
Figure 3 (on next page)

Growth curves distributions.

Average growth curves colored by GL and GS classes. +/- 1 standard error intervals are drawn around the average. A) $G L$ classes. B) GS classes. 


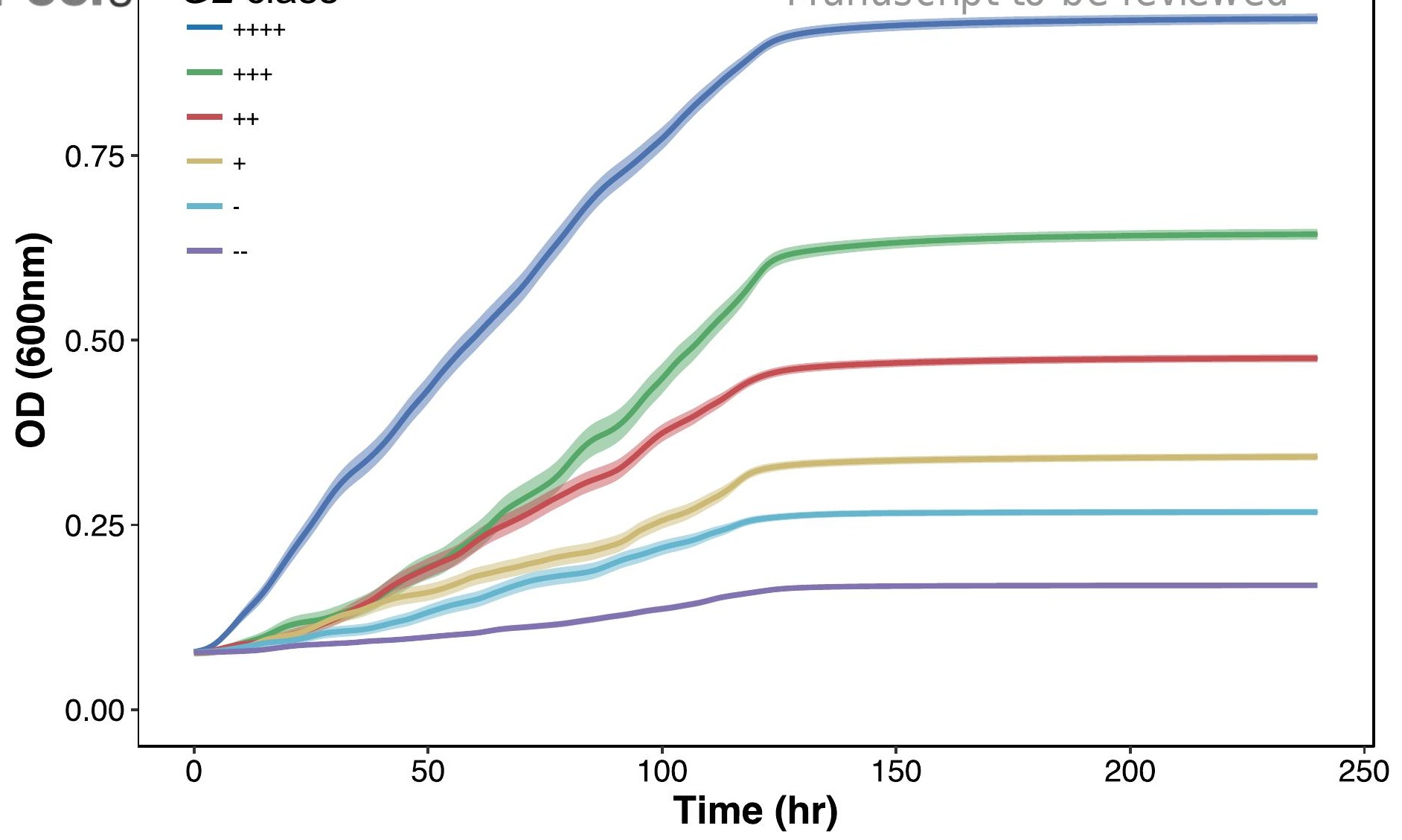

B)

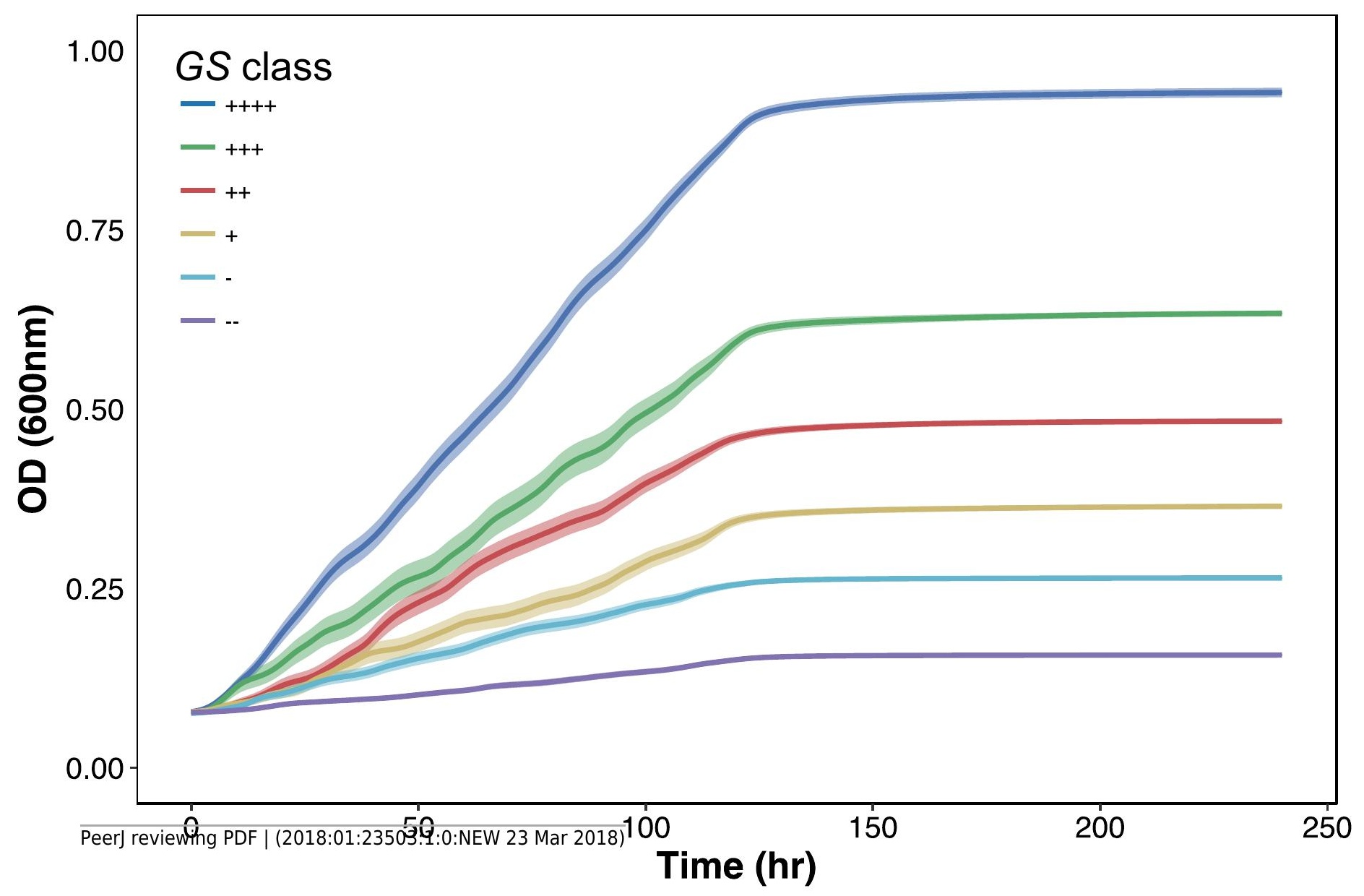


Figure 4 (on next page)

Growth curve parameter distributions.

Box plots presenting the median and interquartile ranges for each simulated growth curve parameter. Data are stratified by $G L$ and GS classes along the columns and by parameters along the rows. Plots demonstrate how each growth metric contains a strong dependence on biomass yield $(A)$ and growth rate $(\mu)$. Lag time and starting yield did not have strong effects on growth class. A) GL classes. B) GS classes. 
A) Growth Level

A)
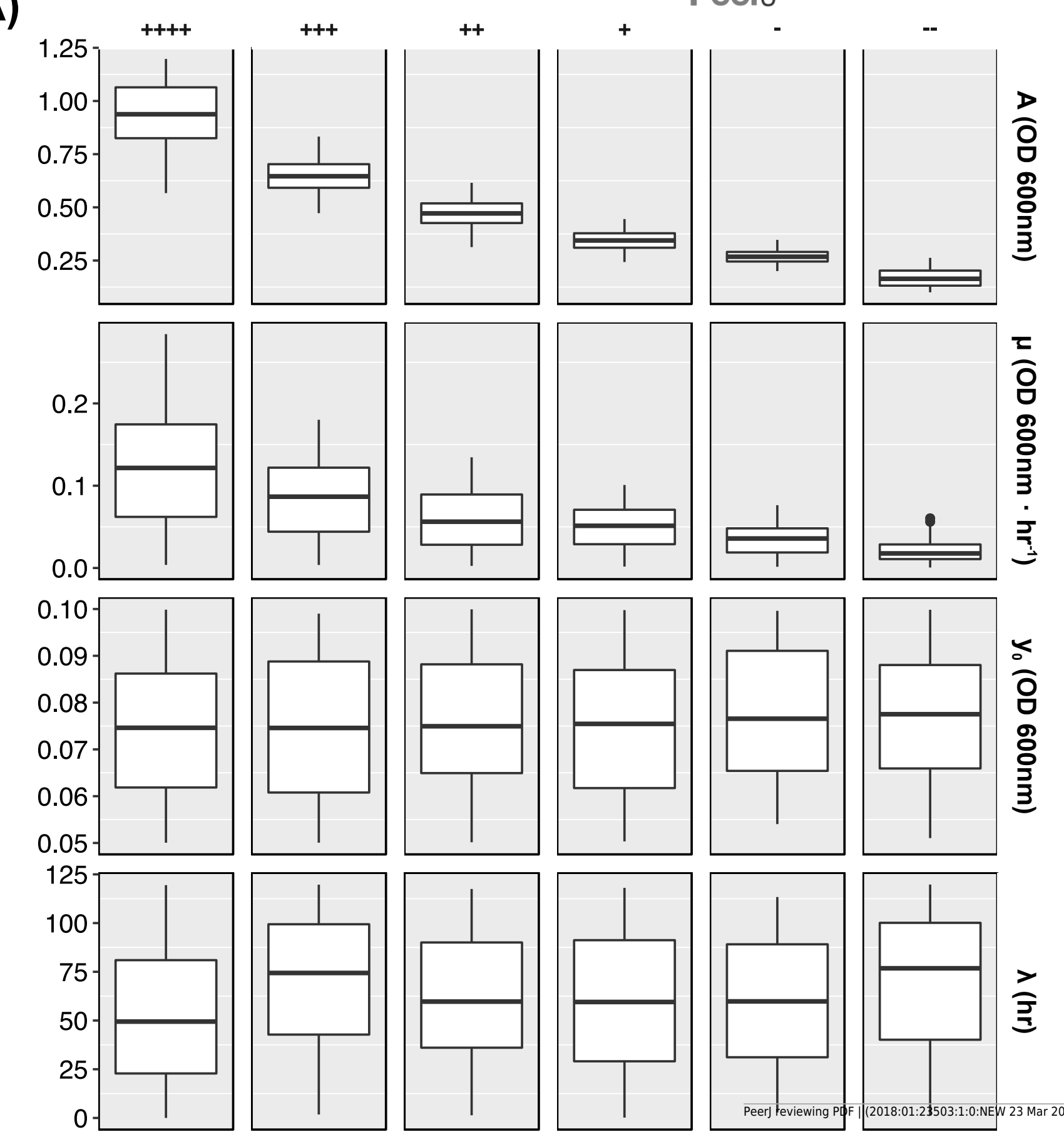
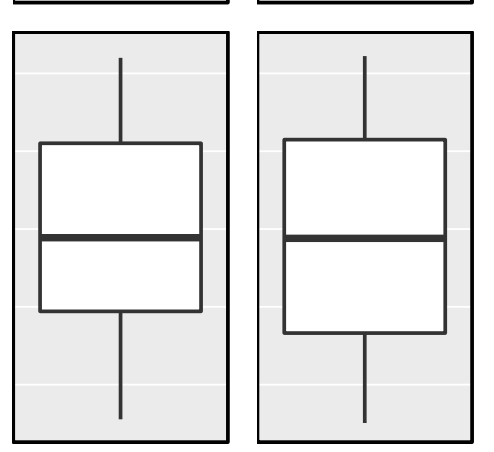

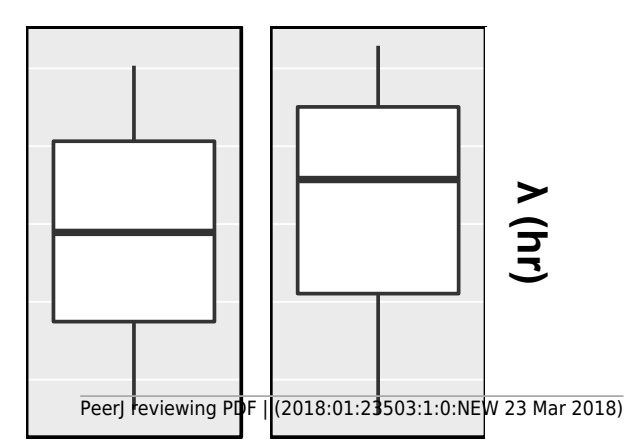

B) anusc Growth Scored

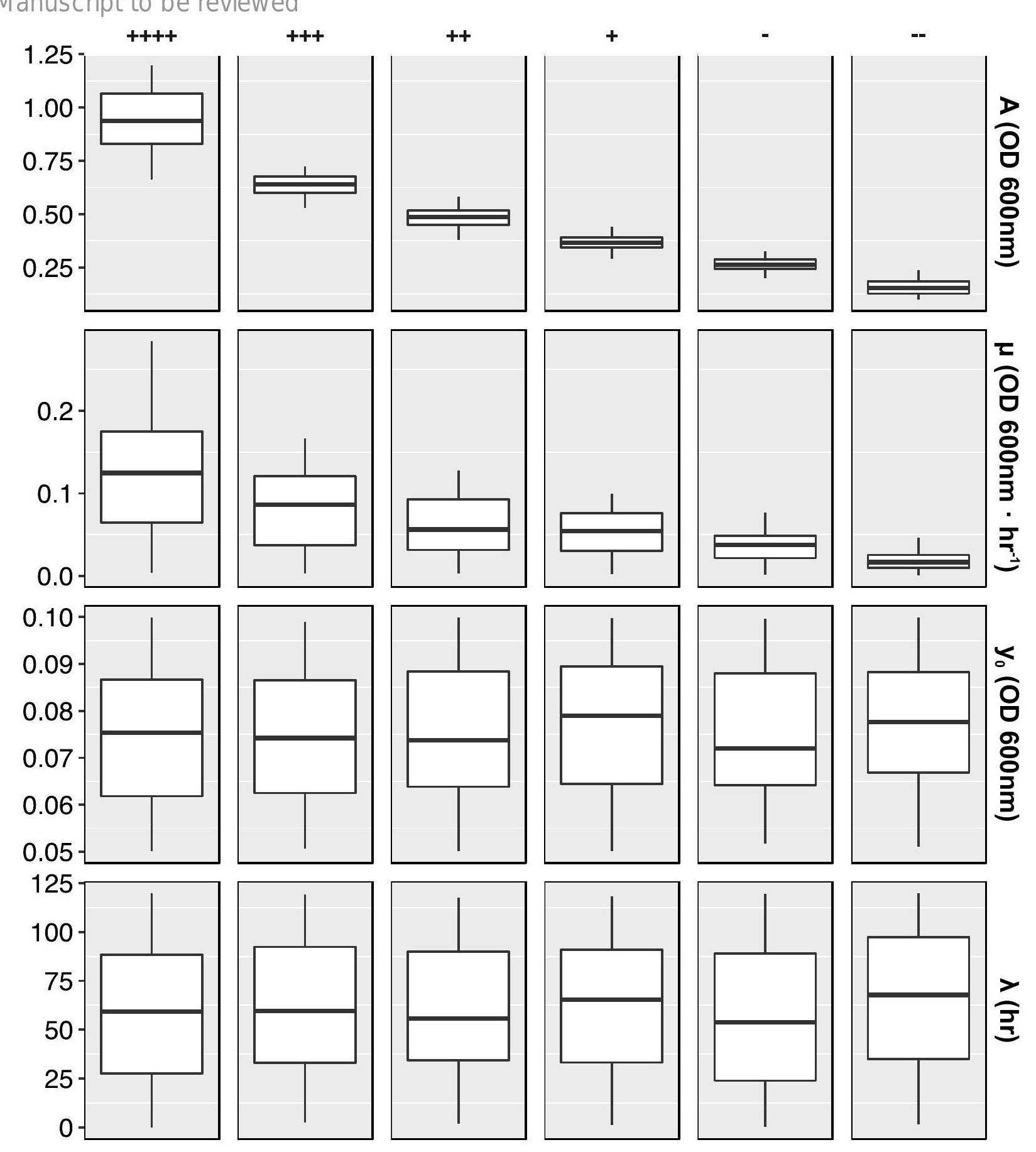

